\title{
AS PERSPECTIVAS DA CULTURA E EXTENSÃO NAS ESCOLAS DE ENFERMAGEM NO BRASIL
}

\author{
Rosalina Aparecida Partezani Rodrigues* \\ Maria Helena Pessini de Oliveira* \\ Maria Lúcia do Carmo Cruz Robazzi*
}

Enfocou-se o desenvolvimento das atividades ou propostas de cultura e extensão universitária em 14 Escolas de Enfermagem do Brasil, utilizando-se um instrumento com seis questões abertas, as quais foram analisadas de forma descritiva. Os dados mostraram que as Escolas de Enfermagem desenvolvem diversas atividades de cultura e extensão universitária, entretanto não são consideradas prioritárias em relação ao ensino e a pesquisa.

UNITERMOS: cultura, extensão universitária, enfermagem.

\section{INTRODUÇÃO}

O tema que ora apresentamos está relacionado com as atividades de cultura e extensão universitária, desenvolvidas por algumas Escolas de Enfermagem do Brasil. Optamos inicialmente, por explanar a respeito do conceito utilizado para cultura e extensão no contexto da Universidade.

De acordo com FERREIRA ${ }^{1}$, cultura é "o ato ou efeito de cultivar, são padrões de comportamento, as crenças, os valores de uma sociedade, é o desenvolvimento de um grupo social; são as atividades e o desenvolvimento intelectual, o saber, a instrução".

\footnotetext{
* Professor Doutor da Escola de Enfermagem de Ribeirão Preto da Universidade de São Paulo.
} 
Termo de significado abrangente, de acordo com MARCOVITCH ${ }^{3}$, "a cultura de uma Universidade deve repousar em premissas éticas como o compromisso social, a busca da excelência e da verdade, o respeito ao indivíduo, o compromisso social e o senso de responsabilidade na aplicação de recursos".

Baseado nesta compreensão entende-se que a Universidade é uma das maiores produtoras de cultura, uma vez que a própria vida acadêmica deve considerá-la como uma implícita dimensão em todas as suas atividades, incluindo-se a pesquisa e o ensino. Dessa forma, o conhecimento obtido pelos profissionais docentes, a nível de pesquisa aplicada ao ensino e a extensão de serviços a comunidade, formam o tripé básico de interação contínua entre a instituição e a sociedade.

Por sua vez, quanto ao termo extensão, FERREIRA ${ }^{1}$ o entende como a transmissão de conhecimento, é "o ato ou efeito de estender, de ampliar, de desenvolver".

FREIRE $^{2}$ complementa, informando que a extensão é compreendida como um ato educativo, é estender os conhecimentos e suas técnicas para transformar o mundo em que os homens estão, de uma certa forma concreta, científica, é transformar, é modificar a cultura.

MARCOVITCH informa que a extensão tem-se configurado desde os seus primórdios, como uma forma de tornar a Universidade mais presente nos projetos nacionais de desenvolvimento e na resposta as demandas sociais.

É através da atividade de extensão, que o homem vai invadir o espaço do outro, superpondo assim, os valores. Através do dialogo, esse processo facilita a transformação. "É sabendo que sabe pouco que uma pessoa se prepara para saber mais. Se tivéssemos um saber absoluto, já não poderíamos continuar sabendo, pois que este seria um saber que não estaria sendo. Quem tudo soubesse já não poderia saber, pois não indagaria" (FREIRE ${ }^{2}$ ). O homem um ser histórico, inserido num constante movimento de procura, faz e refaz constantemente seu saber, sua cultura.

$\mathrm{Na}$ medida em que essa relação do homem se faz no mundo, nas mais diferentes formas, a nível de produção material e intelectual, a extensão permite um confronto de idéias e de conhecimentos com os outros, e nesse confronto é possível ocorrer mudanças.

A Universidade então, como produtora de cultura e extensão, tem como um de seus papéis, a volta para o ensino e a pesquisa; entretanto, é na extensão educativa que os pesquisadores podem observar, refletir e participar das mudanças sociais e políticas do País.

Ainda, conforme MARCOVITCH ${ }^{4}$, a extensão universitária tem como premissas: ser uma credencial de excelência, pois as Universidades conseguem repassar a comunidade externa, em forma de serviços ou ensinamentos, o conhecimento acumulado em todas as áreas; - prestar assistência a população; - cobrir universo significativamente maior que os cursos formais de graduação e pós-graduação; democratizar o saber acadêmico, através de propostas e estudos estratégicos; - situar nos campos de serviços e nos de natureza cultural, questões estratégicas ou pesquisa e defesa da cidadania; - induzir os docentes a tomarem conhecimento das expectativas 
da sociedade, para conciliar, na pesquisa, o rigor metodológico e a relevância social; caracterizar o perfil da Universidade, entendida como instituição a serviço da coletividade.

As enfermeiras e/ou outros profissionais da área da saúde que atuam na docência e pesquisa, preocupados com estas questões, não podem se alijar desse processo de constante movimento, envolvendo um contexto histórico, social e político da sociedade. Para visualizar esta realidade, tivemos como objetivo conhecer quais as atividades ou projetos de cultura e extensão desenvolvidas pelas Escolas de Enfermagem no Brasil e indagamos como as mesmas, enquanto instituições Universitárias estão desenvolvendo esses trabalhos.

\section{FOCALIZANDO A METODOLOGIA DO TRABALHO}

O estudo pretendeu abranger 23 Escolas de Enfermagem do Brasil, dos Estados: Bahia, Ceará, Espírito Santo, Goiás, Minas Gerais, Mato Grosso, Paraíba, Paraná, Pernambuco, Santa Catarina, São Paulo, Rio Grande do Sul e Rio de Janeiro, abrangendo representantes das regiões Norte, Nordeste, Sudeste, Sul e Centro Oeste do país. Destas Escolas, 15 são vinculadas às Universidades Federais, 05 às Estaduais e 03 às Particulares.

Foi elaborado um questionário para essas instituições, com as seguintes questões:

1 - A sua Unidade tem programas de extensão à comunidade?

2 - O que os docentes desta Unidade entendem por cultura e extensão Universitária?

3 - Para o desenvolvimento desse programa, a Unidade recebe verbas? Citar.

4 - Descreva as atividades que são desenvolvidas no programa.

5 - Quais os benefícios que o programa de extensão tem oferecido para a Comunidade?

6 - Quais as dificuldades que a Unidade tem encontrado para o desenvolvimento do programa?

O questionário foi então enviado as 23 Escolas, anteriormente citadas, recebendo-se a resposta de 14 (56\% das instituições selecionadas) representantes de dez Estados da nação.

Os dados foram categorizados e analisados de forma descritiva.

\section{APRESENTANDO E DISCUTINDO OS DADOS}

Verificou-se que todas as 14 Escolas de Enfermagem responderam que desenvolvem programas de cultura e extensão à comunidade, com projetos a nível assistencial, educativo e de pesquisa. 
Pelas respostas obtidas, pode-se perceber que entende-se por projeto assistencial os programas que prestam assistência a saúde, a grupos específicos da população, como: crianças (recém-nascidos e escolares), adolescentes, adultos e idosos, na área preventiva e curativa. Enfoca ainda, aspectos de imunização, gravidez normal, gravidez de alto risco, doenças crônico-degenerativas, cirurgias de cabeça e pescoço, abdominais, ortopédicas e ginecológicas, nas instituições de saúde e nos domicílios.

Quanto aos aspectos educativos, isto é, voltados para a comunidade, estão relacionados a cursos de primeiros socorros, amamentação, educação geral a saúde, reuniões comunitárias com palestras diversas a grupos educativos com alcoólatras, apoio a criança, adultos de modo geral e mulheres, vivências com idosos, dentre outros.

No que se refere à formação de recursos humanos, as Escolas de Enfermagem tem desenvolvido cursos de extensão universitária, de atualização, aperfeiçoamento e de especialização, implementados a partir das necessidades dos profissionais, nas diferentes regiões do país. Nas regiões sul e sudeste os cursos oferecidos estão mais voltados para a área da assistência hospitalar, buscando o atendimento individualizado enquanto que nas regiões norte, nordeste e centro oeste voltam-se prioritariamente para o interesse comunitário.

A atividade voltada para a pesquisa na sua maior parte relaciona-se com os projetos de extensão universitária, considerando que algumas pesquisas são realizadas para a avaliação dos referidos projetos.

No item "o que se entende por cultura e extensão?", percebe-se, pelas respostas enviadas, que há divergência entre os conceitos emitidos: prioritariamente, as respostas se referem às questões de extensão, mas a sua essência permanece a mesma. Algumas respostas encontram-se a seguir: "é a interação sistematizada da Universidade com a comunidade, prestação de serviço, contribuindo com o desenvolvimento da comunidade, visando troca de experiências e conhecimentos, fortalecendo o ensino e a pesquisa"; "a cultura e a extensão articulam o ensino e a pesquisa, viabilizando a relação transformadora entre a Universidade e a sociedade".

No parecer da Universidade, os programas de extensão universitária resultam em modificações nas condições de vida da comunidade; é através da participação efetiva dos docentes com a população que há troca a do conhecimento científico e popular. A Universidade tem o compromisso de compartilhar seu conhecimento produzido com a comunidade, ou seja, ela estende a cultura produzida em seu âmago, à sociedade.

Quanto às verbas que contemplam os diversos projetos, a grande maioria das instituições participantes do trabalho as recebe da própria Universidade, dos Governos Municipais, Estaduais e Federais, Organização Holandesa para Cooperação Internacional de Desenvolvimento, fundação Interamericana, Organização Pan-americana de Saúde, Conselho Nacional de Desenvolvimento Científico e Tecnológico (CNPq) e diversas Fundações.

Em relação à descrição das atividades nos programas, foi mencionado que há uma evidente e ativa participação da comunidade nos diversos projetos universitários, 
valorizando por sua vez, os acadêmicos, o saber popular, visando a melhoria da qualidade de vida das pessoas, bem como no resgate da sua cidadania. Parece existir também uma certa organização da sociedade e diversas articulações com a Universidade. Os programas também favorecem a comunidade, o crescimento da organização política de grupos da população; outros benefícios estão relacionados com os programas voltados para o atendimento das situações de saúde e de doença. Entre esses, pode-se citar: programas educativos, preventivos, curativos e de reabilitação as crianças, adolescentes, adultos e idosos, enfocando aspectos voltados para o aleitamento materno, saúde escolar, pré-natal, parto, puerpério, aos ostomizados, as mastectomizadas, hipertensos, diabéticos, aidéticos, saúde do trabalhador, diversos grupos de vivência, principalmente com aposentados, dentre outros.

No que tange aos benefícios para a formação de recursos humanos, as Escolas de Enfermagem participantes tem oferecido oportunidades aos profissionais da enfermagem de se atualizarem em diversas modalidades de ensino. Os temas têm sido os mais diversos, abrangendo assistência individual ou coletiva. Há a abertura dessas instituições para os profissionais se atualizarem e os mesmos buscam espaço para discussão dos problemas, desenvolvimento da critica sobre a realidade, incentivando transformações.

O serviço de extensão universitária tem beneficiado os docentes, pois thes oferece entrosamento com diversas associações, visando o conhecimento da situação de saúde da população.

A respeito das dificuldades nos diversos projetos de extensão universitária evidenciase: falta de recursos humanos, materiais, financeiros e sobrecarga dos professores com atividades de ensino e pesquisa, número de docentes insuficientes. Além disso, os relatos das Escolas de Enfermagem mostram que a extensão encontra-se visivelmente em patamares secundários na escala de valores acadêmicos, não tendo o mesmo peso que o ensino e as atividades de pesquisa. Ainda como dificuldades, as Escolas colocaram que há necessidade de se rediscutir as atividades de cultura e extensão universitária, pois há falta de uma definição adequada na política Universitária, quanto a essas atividades; a infraestrutura é inadequada; existe pouco envolvimento e, portanto, há necessidade de maior integração institucional; há falta de compromisso dos docentes, de apoio administrativo, de espaço físico apropriado e existe uma demanda elevada dos serviços de extensão em relação ao número de professores para realizá-los.

\section{CONSIDERAÇÕES FINAIS}

Podemos perceber, pelo exposto, que as Escolas de Enfermagem brasileiras despendem um razoável esforço, no sentido de realizarem atividades de cultura e extensão, priorizando a extensão universitária.

Através dos diversos projetos de extensão universitária há produção de diversas 
pesquisas, o que demonstra um certo deslocamento da cultura produzida para o exterior da Universidade. Os profissionais que atuam em diversas instancias institucionais, bem como outros elementos da comunidade em geral, recebem informações das mais variadas, de acordo com suas necessidades. Os programas são do tipo assistenciais e/ou preventivos, realizados em nível hospitalar e/ou domiciliar, percorrendo as unidades básicas de saúde e atendem crianças, adolescentes, adultos e idosos, de ambos os sexos, doentes ou saudáveis, com níveis diversos de necessidades.

Os cursos dados os profissionais abrangem as diversas modalidades, do tipo especialização, aperfeiçoamento, extensão universitária, difusão cultural, e proporcionam reciclagem permanente aos enfermeiros de serviço que, por sua vez, repassam esse conhecimento a sua clientela.

Varias dificuldades foram apontadas, as quais prejudicam os programas a comunidade, tais como: falta de recursos humanos e/ou materiais, falta de verbas, equipamentos, docentes não motivados para realizar atividades de cultura e extensão, dentre outros. Assim, existe uma relativa "pouca" ressonância dentro da própria Universidade, em relação a essas atividades, que não são valorizadas no mesmo patamar que as de pesquisa, e as de ensino de graduação e pós-graduação.

Podemos visualizar que essas dificuldades refletem a realidade da sociedade que não estabelece metas para o processo critico da educação. Também essa mesma sociedade, beneficiária por excelência dos serviços de extensão, provavelmente tenderá a não considerá-los como prioritários, não tão legítimos como os demais. Há necessidade de rediscutir em todo o Brasil, a política universitária, nos que tange aos programas de cultura e extensão, não apenas nas Escolas de Enfermagem, mas em todos os segmentos da Universidade. Para o planejamento e implementação dessa extensão é necessário uma legitima articulação com a comunidade e uma real participação do resgate social, para que possa ocorrer a ampliação deste papel da Universidade, aumentando então, a academia, o seu conhecimento, através da contribuição oferecida pela sociedade, e repassando o seu saber acadêmico, garantindo assim uma legítima e saudável bilateralidade.

\section{CULTURE AND UNIVERSITY EXTENSION PERSPECTIVES AT THE COLLEGES OF NURSING IN BRAZIL}

The present study focused the development of activities or cultural proposals and university extension in 14 Brazilian Nursing Schools, utilizing an instrument with six open questions that were analyzed in a descriptive way. Data showed that Nursing Schools develop several cultural and university extension activities. However, these activities are not considered priority in relation to teaching and research.

UNITERMS: culture, university extension, nursing. 


\section{LAS PERSPECTIVAS DE LA CULTURA Y EXTENSIÓN UNIVERSITARIA EN LAS ESCUELAS DE ENFERMERÍA EN BRASIL}

Se enfocó el desarrollo de las actividades o propuestas de cultura y extensión universitaria en 14 escuelas de Enfermería en brasil, utilizándose un instrumento con seis cuestiones abiertas, las cuales fueron analizadas en forma descriptiva. Los datos mostraron que las Escuelas de Enfermería desarrollaron diversas actividades de cultura y extensión universitaria, entre tanto, no son consideradas prioritarias con relación a la enseñanza y la investigación.

UNITERMOS: cultura, extensión universitaria, enfermería.

\section{REFERÊNCIAS BIBLIOGRÁFICAS}

01. FERREIRA, A.B. de H. Novo dicionário da língua portuguesa. 2. ed. Rio de Janeiro: Nova Fronteira, 1986.

02. FREIRE, P. Extensão ou comunicação? 5. ed. Rio de Janeiro: Paz e Terra, 1980.

03. MARCOVITCH, J. Diretrizes sobre cultura na USP/ Apresentado ao Conselho de Cultura e Extensão Universitária, da Pró-Reitoria de Cultura e Extensão Universitária da USP, 10 de março 1994. p. 4 (mimeografado).

04. Diretrizes para a Extensão Universitária na USP/ Apresentado ao Conselho de Cultura e Extensão Universitária, da Pró-Reitoria de Cultura e Extensão Universitária da USP, 5 de maio 1994, p. 4. (mimeografado). 